\title{
EPOS - Das elektronische Sprachenportfolio. Entwicklung, Didaktik, Perspektiven
}

\author{
Jürgen Friedrich † (Bremen/Deutschland)
}

\section{Einleitung (Bärbel Kühn)}

Das Elektronische Portfolio der Sprachen, EPOS, ist eine Entwicklung des „Runden Tisches Sprachen“ in Bremen, das zuerst an ausgewählten Bremer Schulen erprobt wurde. Die Einführung am Sprachenzentrum der Hochschulen im Land Bremen (FZHB) übernahm ich als dessen Geschäftsführerin und setzte es v.a. im Selbstlernbereich an der Universität ein. Dazu arbeitete ich nicht nur mit den Mitarbeiter*innen des FZHB zusammen, sondern auch mit dem Informatiker Jürgen Friedrich von der Universität Bremen. Er entwickelte EPOS technisch weiter und engagierte sich nach seiner Emeritierung gemeinsam mit mir, u.a. in einem Projekt des Europäischen Sprachenzentrums des Europarats, für die Verbreitung von EPOS in Europa. Jürgen Friedrich starb im August 2018. Wir konnten die uns vorgelegte erste Version seines Beitrags nicht mehr mit ihm diskutieren, haben ihn nur leicht gekürzt und veröffentlichen ihn hier zu seinem Gedächtnis.

\section{Autonomes und handlungsorientiertes Lernen}

Spiegelt man die Didaktik des Einsatzes von ePortfolio-Systemen an den pädagogischen Theorien des Lernens, dann sind vor allem zwei Elemente von grundsätzlicher Bedeutung: Der Ansatz des autonomen Lernens und das Konzept der Handlungsorientierung. Dabei schließt Handlungsorientierung die Kategorie des Kontextes ein. Niemand handelt im luftleeren (heißt: gesellschaftsleeren) Raum, alles ist situativ. 
Lernerautonomie in einem umfassenden Sinne bezieht sich einerseits auf die eigenständige Wahl von Lernstrategien, Lernmethoden, (alternativen) Lösungswegen und Ressourcen zur Unterstützung des Lernprozesses (prozessuale Autonomie). Andererseits meint sie die selbstbestimmte Auswahl von Lernzielen, Lerngegenständen und Lösungsansätzen (inhaltliche Autonomie). Der häufig mit dem Begriff des autonomen Lernens insgesamt gleichgesetzte Begriff des selbstgesteuerten Lemens wird im Rahmen dieses Beitrags enger gefasst und entspricht eher dem oben genannten Teilaspekt der prozessualen Autonomie, bei der die Lernziele im Wesentlichen vorgegeben sind. Insofern kann man sagen, dass etwa „lebenslanges Lernen“ im Verhältnis zum institutionellen Lernen mit seinen allgegenwärtigen „Rahmenplänen“ einem ganzheitlicheren Begriff von Autonomie folgt.

Die genannte Wahlfreiheit der Lernenden ist nur die eine Seite der Lernerautonomie. Ihr entspricht auf der anderen Seite die Eigenverantwortlichkeit und die Eigeninitiative im Lernprozess. Die Gewissheit der „alten Schule“, dass die Institution und die Lehrenden schon die Qualität des Lernprozesses sicherstellen, wird durch die Reflexion des eigenen Lernprozesses ersetzt (Metakognition). Der/die Lernende versichert sich dadurch selbst der (mehr oder weniger erreichten) situativen Qualität. In der Fremdsprachendidaktik spielt das Konzept der Lernerautonomie eine herausragende Rolle (vgl. z.B. Holec 1981, Little 1991, Tassinari 2010). Schon in dem vor 30 Jahren begonnenen Entwicklungsprozess des Gemeinsamen europäischen Referenzrahmens für Sprachen war Autonomie eine zentrale Kategorie, wie weiter unten ausgeführt wird.

Autonomes Lernen ergibt sich bei den Lernenden nicht „,von selbst“. Autonomie ist mühsam, so ähnlich wie Demokratie mühsam ist. Viele Lernende sind Autonomie nicht gewöhnt, manche scheitern an ihr. Daher muss autonomes Lernen ${ }^{1}$ selbst gelernt werden und bedarf dabei der Anleitung und Begleitung durch die Lehrenden. Die Rolle der Lehrenden wiederum wandelt sich von der reinen Wissensvermittlung zur Beratung, zum Empowerment. Insofern stellt autonomes Lernen auch für die Lehrenden eine neue Herausforderung hinsichtlich ihres Selbstverständnisses und ihrer Kompetenz dar. Nach dem hehren Postulat des autonomen Lernens folgen die Mühen der Ebene.

Folgt man der Annahme, dass Lernen in den allermeisten Fällen nicht dem reinen Wissenserwerb dient, sondern Handlungsfähigkeit in einem bestimmten Kontext (Kompetenz) zum Gegenstand hat, dann leuchtet unmittelbar ein, dass diese Handlungsfähigkeit nicht (allein) aus Wissen resultiert - selbst wenn dieses Wissen in einen Kontext eingebettet ist. Kompetenz, eben auch sprachliche Kompetenz, erwirbt man durch Tun. Insofern ist spätestens seit der Entstehung der Reformpädagogik (vgl. z.B. Dewey 1916) klar, dass Handlungsfähigkeit nur durch

\footnotetext{
${ }^{1}$ Entsprechend Holecs berühmter Definition: Autonomous learning is ,the ability to take charge of one's own learning“" (Holec 1981: 3).
} 
Handeln erzielt werden kann. ${ }^{2}$ In der Pädagogik ist das Primat des handlungsorientierten Lernens heute weitgehend anerkannt und wissenschaftlich begründet, ${ }^{3}$ auch deshalb, weil die Handlungsanforderungen in der modernen Lebens- und Arbeitswelt - einschließlich der sprachlichen und allgemein kommunikativen - zunehmend komplexer werden und daher nicht mehr durch die einfache Anhäufung von Wissen gelöst werden können. Das bedeutet nicht, dass Wissensvermittlung unwichtig wäre, aber sie muss eingebettet sein in eine auf Kompetenzen gerichtete Lehr-Lernsituation.

\section{Portfolioarbeit und der Europäische Referenzrahmen}

Die Sprachlehrforschung und die Praxis der Sprachvermittlung haben dies frühzeitig erkannt, was dazu führte, dass im Rahmen der Erarbeitung des Gemeinsamen Europäischen Referenzrahmen für Sprachen (Europarat 2001) die Nutzung eines Portfolios für die pädagogische Umsetzung früh mitgedacht wurde.

\subsection{Portfolioarbeit als didaktischer Ansatz}

Portfolios dienen in der Pädagogik einerseits der Sammlung von Lernergebnissen, andererseits aber ganz wesentlich auch als Mittel zur Unterstützung und Reflexion dieser Ergebnisse und des eigenen Lernprozesses. ${ }^{4}$ Es geht also nicht in erster Linie um „Lernen aus Büchern“ oder „,on Lehrenden“, sondern um das „eigene Tun“. Basis der Portfolio-Didaktik sind damit handlungstheoretische und konstruktivistische Ansätze, die von einem ergebnisoffenen, die Lernenden herausfordernden und weitgehend selbstbestimmten Lernprozess ausgehen. Punktuelle Ergebnisbewertungen werden ergänzt durch die Dokumentation und Bewertung des kontinuierlichen Kompetenzerwerbs, der Lösungsansätze und Lernmethoden. Neben die Fremdeinschätzung der Leistungen durch die Lehrenden tritt die Selbsteinschätzung der Lernenden, neben die Beurteilung als Aufgabe der Lehrenden tritt die Beratung, die die Defizite des gegenwärtigen Leistungsstandes eher als Ausgangspunkt zur Nutzung von Chancen im weiteren Lernprozess begreifen (vgl. Reich o.J., Brunner et al. 2008). Darüber hinaus bieten Portfolios die Möglichkeit zur Differenzierung. Lerner*innengruppen sind heute in Bezug auf berufliche Vorerfah-

\footnotetext{
2 Eigentlich ist das Konzept des handlungsorientierten Lernens wesentlich älter als die Reformpädagogik, siehe etwa Dewey „Learning by doing“. Manche führen es auf Aristoteles zurück, aber spätestens seit Comenius (1592-1670) ist „Lernen durch Tun“ eine didaktische Grunderkenntnis (Comenius 2008/1657).

3 Vgl. z.B. die ausführliche Bibliographie zu autonomem Lernen von Hayo Reinders (o.J.).

${ }^{4}$ Das ist nicht selbstverständlich, wenn man sich etwa die Definition des European Institute for E-Learning ansieht: „An ePortfolio is a personal digital collection of information describing a person's learning, career, experience, and achievements“ (zit. nach Emmett 2011: 6). Hier steht das „Konzept Sammelmappe" noch stark im Vordergrund; Interaktion und Reflexion spielen keine Rolle.
} 
rung, kulturellen Hintergrund, Lernerfahrung, Motivation zunehmend heterogen zusammengesetzt (vgl. Buschmann-Göbels \& Jahnke 2017: 180). Damit ist eine einheitliche „Beschulung“ aller Kursteilnehmer*innen nicht mehr zielführend. Portfolios ermöglichen die individuelle Beratung, Förderung und Beurteilung einzelner Lernender ausgehend von ihrem Lernstand und Lernkontext.

Portfolios gibt es in ganz unterschiedlichen Ausprägungen, wobei diese in der Praxis oft auch als Mischformen anzutreffen sind (vgl. Egle 2015). Um nur einige zu nennen:

- Prozessportfolios (auch: Entwicklungsportfolios) sind weniger an Ergebnissen orientiert, sondern zeigen die Landschaft auf, die eine Lernerin/ein Lerner durchlaufen hat, und reflektieren die Wege, Umwege und Auswege des Lernprozesses. Sie können Selbsteinschätzungen und Arbeiten vom Anfang und solche vom Ende des Lernwegs enthalten, um den Lernfortschritt vergleichend sichtbar zu machen. Wenn die Reflexion zum Hauptkriterium eines Prozessportfolios wird, sprechen wir auch von einem Reflexionsporffolio.

- Produktportfolios dienen der Sammlung von Produkten (Lernergebnissen) einer und eines Lernenden, oft zum Nachweis der Erbringung bestimmter Leistungen. Präsentationsporffolios dienen der Entwicklung und Darstellung von Lern- oder Arbeitsergebnissen (und ggfs. auch -prozessen) für andere. Dies können Mitstudierende, Lehrende oder etwa auch Konferenzteilnehmer*innen sein.

- Bewerbungsporffolios sind für die Vorlage bei einer Entscheidungsträgerin/ einem Entscheidungsträger bestimmt. Das kann das Zulassungsgremium eines Studiengangs sein oder auch ein potenzieller Arbeitgeber/eine potentielle Arbeitgeberin.

- Prïfungsporffolios (auch: Beurteilungsportfolios) dienen der Leistungsbewertung und müssen daher - anders als dies bei den an Offenheit orientierten Prozessportfolios der Fall ist - auf klare Beurteilungskriterien, Lernzielkataloge oder Curriculumsanforderungen bezogen sein und Vergleichbarkeit gewährleisten.

Für das Sprachenlernen und den Einsatz von EPOS spielen vor allem das Prozessund das Präsentationsportfolio eine wesentliche Rolle. ${ }^{5}$

\footnotetext{
${ }^{5}$ Von Lehrenden wird aber oft die Frage aufgeworfen, ob EPOS auch als Prüfungsportfolio eingesetzt werden kann. Darauf kann in diesem Beitrag nicht weiter eingegangen werden (siehe dazu z.B. Häcker 2011).
} 


\subsection{Der Gemeinsame Europäische Referenzrahmen für Sprachen (GER)}

Die Sprachlehre war eine der ersten Disziplinen, in denen ein kompetenzorientierter Ansatz breitere Beachtung fand. Vorreiter bei der Initiierung und Implementierung dieses Ansatzes war (und ist bis heute) der Europarat und sein Language Policy Programme. Alles begann 1991 mit einem Symposium in Rüschlikon (Schweiz) mit dem Titel „Transparenz und Kohärenz im Sprachenlernen in Europa: Ziele, Evaluation, Zertifizierung“. Es ging um eine Antwort auf die zunehmende Mobilität, Migration und internationale Kommunikation in den europäischen Ländern und in Sonderheit um die Entwicklung eines gemeinsamen Instrumentariums, das in allen Ländern als Referenz genutzt werden konnte, ohne dabei die kulturelle Identität und Vielfalt in den Ländern und Regionen außer Acht zu lassen. Dieser Referenzrahmen (Europarat 2001) sollte die Kooperation zwischen den Bildungseinrichtungen und die gegenseitige Anerkennung sprachlicher Kompetenzen unterstützen. Dabei wurde Sprachfähigkeit immer schon als Mehrsprachigkeit und als Teil einer umfassenden (inter-)kulturellen Kompetenz verstanden. Damit waren die Kernbegriffe des GER definiert: Handlungsorientierung, Plurilingualität und Plurikulturalität.

Nach mehr als zehn Jahren Erfahrung mit dem GER begann 2014 dessen Überarbeitung und Ergänzung, die 2017 mit der Formulierung eines „Companion Volume“ abgeschlossen wurde (Council of Europe 2018a). Die mehr oder weniger rein linguistischen Kompetenzbereiche des ursprünglichen GER - einerseits rezeptive Sprachfähigkeiten (Leseverständnis, Hörverständnis) und andererseits produktive Kompetenzen (Sprechen und Schreiben) - sollten um die Fähigkeit, zwischen Menschen sprachlich vermitteln zu können (Mediation) ergänzt werden. Zwar war mit der Kompetenz „Miteinander sprechen“ bereits ein dialogisches Element im ursprünglichen GER angelegt, dabei spielte der Aspekt der kulturellen Kompetenz, die notwendig ist, damit ein solcher Mediationsprozess zwischen Sprachen - und das heißt zwischen Kulturen - gelingen kann, aber noch eine untergeordnete Rolle, und die Fähigkeit zur Vermittlung zwischen Gesprächspartner*innen war noch vollständig ausgeblendet.

Neben der Mediation wurden weitere Kommunikationssituationen ergänzt, z.B. wurden Deskriptoren für Online-Kommunikation und -Interaktion, für Zeichensprachen oder auch für Mehrsprachigkeit entwickelt. Darüber hinaus ging es um die Erweiterung der Sprachlernmethoden, z.B. durch Ergänzung von Ansätzen zum kollaborativen Sprachenlernen. Die neue Fassung des GER greift aber noch wesentlich weiter, indem sie einen Brückenschlag zu anderen Schlüsselkompetenzen herstellt. So wurde bereits ein dreibändiger Referenzrahmen zu Kompetenzen für demokratische Kultur erarbeitet und in Deskriptoren operationalisiert (Council of Europe 2018b, Europarat 2018). 


\subsection{Das Europäische Sprachenportfolio}

Das im GER vertretene Konzept eines Kompetenz- und handlungsorientierten Sprachenlernens legt die Nutzung eines Portfolios zur methodischen Unterstützung ausgesprochen nahe. Entsprechend wurde dieser Ansatz bereits in Rüschlikon diskutiert (Council of Europe 1992) und mit der Etablierung des Europäischen Sprachenportfolios (ESP) einige Jahre später umgesetzt (Council of Europe 2006).

Der ESP besteht aus drei Teilen, dem Sprachenpass, der Sprachenbiographie und dem Dossier. Der Sprachenpass beschreibt die aktuellen Kompetenzen der Lernenden hinsichtlich verschiedener Sprachen mit Bezug zu den sechs Niveaustufen des GER. ${ }^{6}$ In ihm können formale Qualifikationen, aber auch sprachliche und interkulturelle Erfahrungen dargestellt werden. Dabei soll angegeben werden, ob die Feststellung der Qualifikationen auf Selbsteinschätzung oder auf der Beurteilung durch Lehrende beruht. Die Sprachbiographie dient der Planung, Bewertung und Reflexion des eigenen Lernprozesses und des Lernfortschritts, und sie definiert Lernziele für die weitere sprachliche Entwicklung einer oder eines Lernenden. Im Dossier schließlich kann die/der Lernende ihre/seine Lernergebnisse mit Bezug zu ihrem/seinem Sprachenpass und ihrer/seiner Sprachbiographie dokumentieren. Der GER enthält nur eine Reihe von Beispieldeskriptoren zur Einschätzung der Sprachkompetenz. Er ermutigt Bildungseinrichtungen, die sich der Sprachvermittlung widmen, eigene Versionen von Europäischen Sprachportfolios für unterschiedliche Adressatengruppen zu entwickeln, sei es für einzelne Altersstufen oder Bildungsniveaus, sei es für unterschiedliche Lebenssituationen (z.B. Migration, Erwachsenenbildung) oder berufliche Kontexte, und diese von einem Validierungsausschuss des Europarats hinsichtlich ihrer Konformität mit dem GER überprüfen zu lassen. So entstanden zwischen 2001 und 2010 ca. 120 validierte Europäische Sprachenportfolios (Council of Europe o.J.).

Von der im GER noch deutlich vorhandenen Dimension der Inter- oder Plurikulturalität ist - so beschreibt es David Little in seinem Rückblick auf die Entwicklung des ESP (vgl. Little 2012) - im Sprachenportfolio nur noch wenig erkennbar. Kultur wurde zunehmend von Sprache getrennt, obwohl beide in vielen Fällen eng zusammengehören: in der Literatur, bei sprachlichen Konventionen oder bei kulturell geprägten Gesten, die Sprache begleiten. Aleksandra Sudhershan fasst das Problem so zusammen:

The European Language Portfolio (ELP), as a tool designed to promote learner autonomy and intercultural language learning, has the potential to support learners in the development of such responsibility, although until recently this aim was not realized due to the underdevelopment of the ELP's intercultural component, particularly its self-assessment grid (Sudhershan 2014: 150).

\footnotetext{
${ }^{6}$ Dieser Bezug auf die Niveaustufen bildet die eigentliche Verbindung zwischen ESP und GER.
} 
Der Europarat hat schließlich wegen dieser Defizite des ESP ein eigenständiges Instrument, die „Autobiography of Intercultural Encounters“, entwickelt (Little 2012: 15).

Entwickler*innen und Nutzer*innen des ESP brachten in verschiedenen Seminaren zum Ausdruck, dass das Sprachportfolio ohnehin bereits sehr komplex sei und die Einbeziehung plurilingualer und insbesondere interkultureller Aspekte nur schwer oder gar nicht umsetzbar sei.

Im Übrigen sind aufgrund der traditionellen Lehrmethoden, der ubiquitären Curriculumanforderungen und der gegebenen organisatorischen Restriktionen Vorbehalte gegenüber dem ESP ohnehin erwartbar und kritische Fragen vorprogrammiert: Wie kann garantiert werden, dass die im Dossier hinterlegten Dokumente tatsächlich von den jeweiligen Lernenden stammen? Sind Selbsteinschätzungen der Sprachniveaus durch die Lernenden zuverlässig, und wie kann das Portfolio mit den allseits vorhandenen Prüfungsanforderungen in Verbindung gebracht werden?

Man wird sehen, ob die Aktualisierung und Erweiterung des GER, die sich mit der zentralen Kategorie der Mediation ein genuin kulturbezogenes Thema vorgenommen und dazu auch bereits beispielhafte Deskriptoren entwickelt hat, in ihrer Umsetzung in das ESP die genannten Defizite reduzieren kann.

\section{EPOS - das elektronische europäische Sprachenportfolio}

\section{1 ePortfolio-Systeme in der Sprachlehre}

Die Entwicklung und Nutzung elektronischer Sprachenportfolios liegt schon aus praktischen Gründen nahe: Die klassischen Papierportfolios zum Sprachenlernen, wie sie von den Verlagen bis heute publiziert werden, sind unflexibel. Entsprechend wurden sehr bald nach der Veröffentlichung des GER und des ESP Versuche unternommen, elektronische Medien, offline oder online, für die Umsetzung des ESP zu nutzen. Die Ansätze lassen sich folgendermaßen klassifizieren:

Die meisten der zum Sprachenlernen tatsächlich eingesetzten ePortfolio-Systeme der 1. Generation (2000-2010) wurden bereits in Fehse et al. (2011, Abschnitt 2.2), beschrieben. Zusammengefasst kann man sagen, dass sie überwiegend die statische, auf die Lernergebnisse gerichtete Sammelfunktion und viel weniger die prozessorientierte Seite des ESP umgesetzt haben. Inzwischen sind viele von ihnen auch nicht mehr nutzbar, sei es, weil die Webseiten abgeschaltet sind, oder sei es, weil sie technisch nicht aktuell gehalten wurden und den heute gegebenen technischen Standards nicht mehr entsprechen. 


\subsection{EPOS - Auf den Spuren selbstbestimmten Sprachenlernens}

Im Folgenden werden die Besonderheiten des ePortfolios EPOS beschrieben. Im Sinne von Lernerautonomie als einem wesentlichen Bestandteil des Europäischen Sprachenportfolios ist ihr zentrales Ziel die Förderung selbstbestimmten Sprachenlernens.

\subsubsection{Zur Definition eines sprachenorientierten ePortfolio-Systems}

Geht man von dem didaktischen Postulat der Portfolioarbeit allgemein sowie von den besonderen Anforderungen des Europäischen Sprachenportfolios aus (Kap. 3), so kann man der Entwicklung von EPOS als eines elektronischen europäischen Sprachenportfolios folgende Definition zugrunde legen:

a) Ein ePortfolio-System unterstützt die Formulierung eigener Lernziele und die Organisation eigener Lernprozesse auf der individuellen und der Gruppenebene. Dazu bietet es flexible Instrumente für sprachliche Interaktion, Kommunikation und Kooperation zwischen Peers und mit den Lehrenden an.

b) Ein ePortfolio-System gibt die Möglichkeit, die eigenen Fähigkeiten auf Basis standardisierter Kompetenzraster selbst einzuschätzen und auf Wunsch von anderen einschätzen zu lassen. Kompetenzen können anhand unterschiedlicher Kriterien verglichen werden (z.B. anhand von Zeiträumen, Sprachen oder beurteilenden Personen).

c) Ein ePortfolio stellt Hilfsmittel zur Verfügung, um die Lernfortschritte und die Lerngeschichte einer Nutzerin/eines Nutzers insgesamt zu dokumentieren. Dabei eröffnet es die Möglichkeit zur wiederholten Reflexion der Lernziele, -prozesse und -ergebnisse.

d) Ein ePortfolio-System ermöglicht eine wohlstrukturierte Sammlung von eigenen Dokumenten der/des Lernenden (z.B. Texte, Audiodateien oder Videos), die ihre/seine Kompetenzen, Leistungen und Entwicklungsschritte im Lernprozess aufzeigen.

e) Ein ePortfolio-System ermöglicht es den Lernenden, ein Portfolio für bestimmte Zwecke selbstständig flexibel zusammenzustellen (z.B. für Präsentation, Reflexion oder Bewerbung). Die/der Lernende ist der/die Eigentümer*in des Portfolios und entscheidet selbst darüber, mit wem er/sie es teilt.

\subsubsection{Die Entwicklung von EPOS - wie alles anfing}

Nach der Publikation des Europäischen Sprachenportfolios im Jahr 2000 begannen viele Institutionen mit der Umsetzung in spezifische Sprachenportfolios für bestimmte Bildungssituationen. Das Land Bremen beteiligte sich zusammen mit den Ländern Bayern, Berlin, Brandenburg, Hessen, Nordrhein-Westfalen, Sachsen- 
Anhalt und Thüringen ab 2003 am Verbundprojekt „Sprachen lehren und lernen als Kontinuum" der Bund-Länder-Kommission. In diesem Rahmen wurde ein Sprachenportfolio für die Primarstufe und ein weiteres für die Sekundarstufe I entwickelt und 2007 vom Europarat akkreditiert. Es wurde bereits damals als Webseite publiziert ${ }^{7}$ und stellt einen der frühen Versuche dar, digitale Medien zur Umsetzung des Europäischen Sprachenportfolios zu nutzen. Um die Flexibilität der Medien noch besser zu nutzen, also über den Einsatz von Text- und Audiodateien auch die Interaktivität und Multimedialität (interaktive Aufgaben, Videosequenzen, Podcasts) einzusetzen, wurde das System vom Bremer Landesinstitut für Schule als eigenständige Webanwendung mit dem Namen EPOS (elektronisches Portfolio der Sprachen) weiterentwickelt. Die Zusammenarbeit von Bremer Schulen mit dem Fremdsprachenzentrum der Hochschulen im Land Bremen im Sprachenrat Bremen führte dazu, dass das System auch dort eingesetzt wurde. Da die Performanz des Systems bei gleichzeitigem Einsatz in mehreren Klassen- oder Kursverbänden nicht ausreichte, erfolgte im Jahr 2011 durch das Technologie-Zentrum der Universität Bremen ein Redesign auf Basis eines Open Source ePortfolio-Systems (Mahara). Seit 2013 ist EPOS auch an anderen in- und ausländischen Hochschulen im Einsatz.

\subsubsection{EPOS und die Logik des Sprachenlernens}

Der Aufbau von EPOS folgt der Logik des Sprachenlernens: Zunächst bestimmt man anhand der Niveaus des GER im Rahmen einer Selbsteinschätzung den eigenen Lernstand, z.B. um anschließend die Lernziele festzulegen. Zur Erreichung der Lernziele bearbeitet man im Sinne eines handlungstheoretischen Ansatzes Lernaufgaben oder Projekte. Die Entwürfe, Versionen oder Ergebnisse der Projektarbeit werden im Dossier abgelegt. Danach oder auch zu jedem beliebigen Zeitpunkt im Laufe des Lernprozesses reflektiert man seinen Stand, seine Probleme und Fragen sowie Überlegungen zum weiteren Vorgehen im Lerntagebuch. Eine Beschreibung der längerfristigen Lernperioden, die Erfahrungen der/des Lernenden wie auch die Zertifikate, die das Erreichte nachweisen, können in der Lernbiographie hinterlegt werden. Will man die Elemente des Lernprozesses, seine Reflexion oder seine Ergebnisse mit anderen teilen, dann positioniert man sie in einer frei konfigurierbaren Ansicht, einer Art Poster, und gibt diese anschließend für einzelne Personen oder Gruppen frei.

Dieser Aufbau von EPOS entlang der Chronologie des Sprachenlernens bedeutet nicht, dass der tatsächliche Lernprozess genau diesem Ablauf folgen muss. Im Gegenteil, durch die Anordnung der einzelnen Lernaktivitäten auf unabhängig voneinander benutzbaren Reitern soll die Wahlfreiheit im Lernprozess unterstützt werden. Es gibt keinen „One best way“ des Sprachenlernens.

\footnotetext{
7 http://www.sprachenportfolio.de/Modul1/start.html [01.03.2019].
} 
Auf den ersten Blick sieht die EPOS-Funktionsleiste mit ihren neun Reitern sehr umfassend aus. EPOS bietet aber die Möglichkeit, das Funktionsspektrum an die in einem bestimmten Szenario benötigten Aufgaben anzupassen. Alle nicht benötigten Funktionen können abgeschaltet werden.

In EPOS sind die drei Komponenten des Europäischen Sprachenportfolios vollständig abgebildet, aber die in den ESP-Komponenten oft implizit enthaltenen Vorgaben, z.B. „Festlegung eigener Lernziele“ in der Biographie, sind in EPOS unmittelbar auf der Oberfläche sichtbar. Dadurch soll die Benutzungskomplexität reduziert und ein gewisser „Aufforderungscharakter“ ausgedrückt werden. Abb. 1 macht den Zusammenhang von ESP und EPOS deutlich.

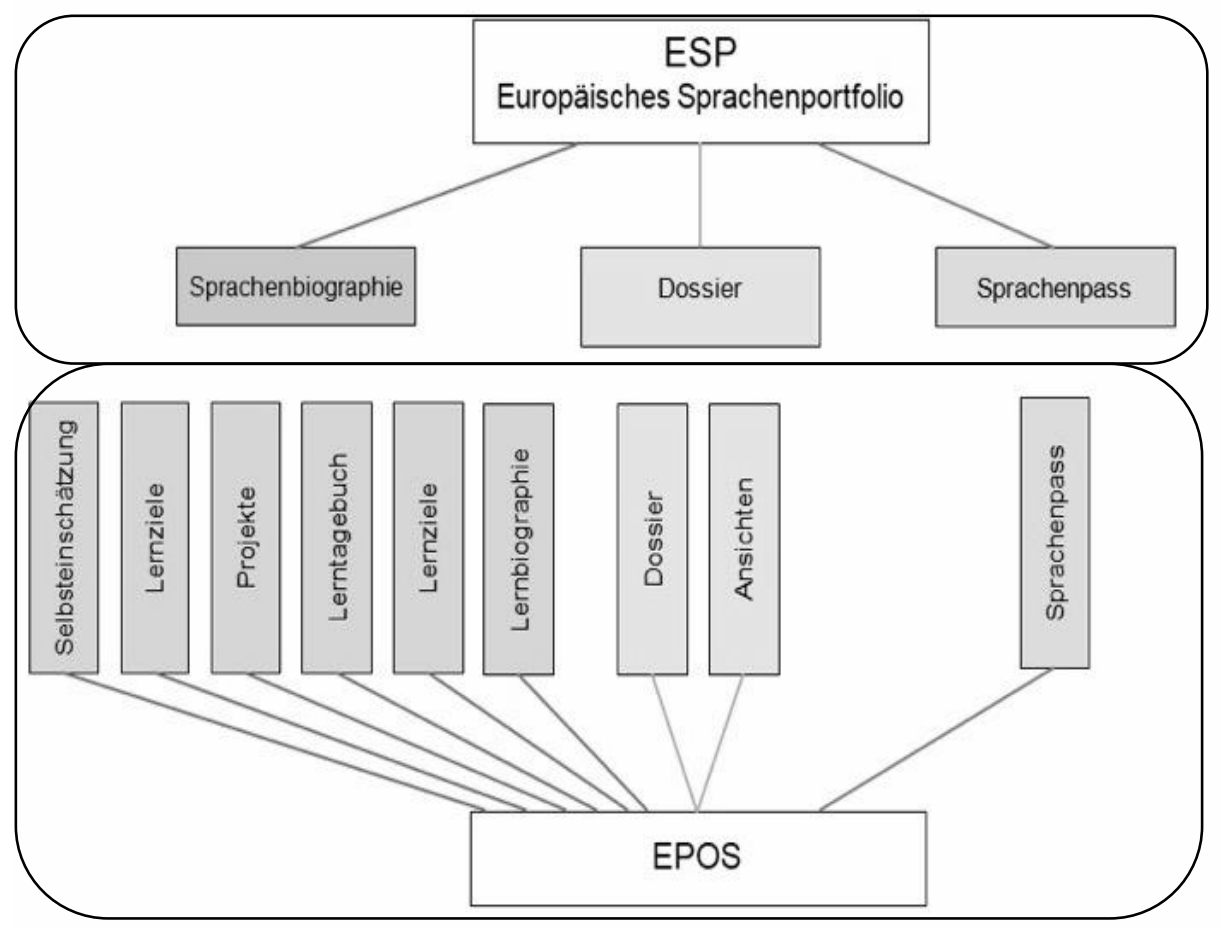

Abb. 1: Zusammenhang zwischen den Elementen in EPOS und den Komponenten des ESP

\subsubsection{Wo EPOS über das ESP hinausgeht}

EPOS bildet nicht nur alle Komponenten des ESP ab, sondern bietet weitere Funktionen, die den ESP ergänzen und bereichern. Die Multimedialität von EPOS erwietert die inhaltlichen und formalen Möglichkeiten des Lernens und könnte die Motivation der Lernenden positiv beeinflussen. Im Folgenden sind einige der zusätzlichen Elemente erläutert. 


\section{Flexible Selbsteinschätzung}

Im traditionellen Sprachenportfolio ist in der Regel eine einzige Deskriptorliste ${ }^{8}$ vorgegeben, die nur nach Niveaustufen gegliedert ist. Die zunehmend vorhandene Diversität der Lernenden erfordert aber eine personale, inhaltliche oder didaktische Differenzierung des (Sprach-)Unterrichts, die durch unterschiedliche Deskriptorlisten innerhalb einer Lernendengruppe unterstützt werden sollte. Ein Beispiel wäre etwa die Nutzung unterschiedlicher Deskriptorlisten in einer Klasse mit Erstund Zweitsprachler*innen, Migrant*innen. In EPOS können der sprachlichen Selbsteinschätzung unterschiedliche Deskriptorlisten zugrunde gelegt werden. Die Deskriptorlisten stammen von Institutionen, die sich intensiv mit Sprachvermittlung beschäftigen. Beispiele sind:

- CercleS (Confédération Européenne des Centres de Langues de l'Enseignement Supérieur; ein europäischer Verband von Sprachenzentren an Hochschulen)

- $\quad$ ELC (European Language Council; ein weiterer europäischer Verband von Sprachenzentren an Hochschulen)

- EAQUALS/ALTE (Evaluation and Accreditation of Quality Language Services/Association of Language Testers in Europe)

- CARAP/FREPA (Cadre de référence pour les approches plurielles/ Framework of Reference for Pluralistic Approaches; ein Projekt des European Centre for Modern Languages - ECML)

Die Kompetenzraster liegen in EPOS in verschiedenen Sprachen vor, zumindest in Deutsch, Englisch und Französisch, teilweise auch in Spanisch und Italienisch. Little (2007) vertritt die Auffassung, dass die Selbsteinschätzung wie auch die Reflexion in der Zielsprache durchgeführt werden sollte. In der Praxis wird diese Frage oft pragmatisch beantwortet: Lernende auf den Niveaustufen A1 und A2 benutzen die Deskriptoren in der Erstsprache, solche auf den Niveaustufen B1, B2 oder C1 können sinnvollerweise die zielsprachlichen Deskriptoren verwenden.

Neben den allgemein linguistisch geprägten Kompetenzrastern existieren in EPOS auch solche, die spezielle sprachliche Anforderungen betreffen, z.B. fachsprachliche Deskriptoren, Deskriptoren, zu besonderen Kommunikationsformen, wie etwa der Online-Konversation und -Kooperation, zu interkultureller Kommunikation, zu Mediation (entsprechend dem GER 2018), zu Mehrsprachigkeit, zur besonderen sprachlichen Situation von Migrant*innen oder zu Sprachlernstrategien.

Die Deskriptoren/Kompetenzen in den Rastern sind oft entweder relativ allgemein oder andererseits auch ziemlich komplex formuliert, wissenschaftlich umfassend und korrekt. In jedem Fall fällt es den Lernenden meist schwer, sich

\footnotetext{
${ }^{8}$ Statt des Begriffs „Deskriptorliste“ wird im vorliegenden Beitrag häufig verallgemeinernd auch der Begriff „Kompetenzraster“ verwendet.
} 
hinsichtlich der so formulierten Kompetenzen selbst einzuschätzen. EPOS bietet zur Abmilderung dieses Problems den Lehrenden die Möglichkeit, die Deskriptoren mit Hilfe von Beispielen oder kleinen Aufgaben zu erläutern. Per Klick können sich die Lernenden bei Bedarf diese Zusatzinformationen anschauen oder durch Lösung der Aufgaben ihre Selbsteinschätzung absichern.

Eine zweite Form der Flexibilität in EPOS bezieht sich auf das Spektrum der möglichen Bewertungen der eigenen Kompetenzen. Das ESP gibt lediglich drei Antwortmöglichkeiten vor: „Ich kann das sehr gut“, „Ich kann das gut“ und „Das fällt mir noch schwer". Lernende haben oft Schwierigkeiten, sich für eine der beiden Kategorien „sehr gut“ oder „gut“ zu entscheiden. EPOS ermöglicht es den Lehrenden daher, Deskriptorlisten mit mehr als zwei Abstufungen zu versehen.

\section{Kooperatives Lermen}

In EPOS gibt es eine Funktion „Gruppe“, die kooperatives Lernen durch die Erstellung gemeinsamer Artefakte und die gemeinsame Reflexion, etwa in Tandems, unterstützt.

\section{Lebenslanges Sprachenlernen}

Der Schwerpunkt der Nutzung des Europäischen Sprachenportfolios liegt in der formalen institutionellen Lehre (Schule und Hochschule), informelles Lernen spielt noch eine geringe Rolle. Das EPOS-Portfolio einer/eines Lernenden kann exportiert (und an anderer Stelle wieder importiert) werden und unterstützt damit den Wechsel zwischen Institutionen, eröffnet aber vor allem die Chance zur Umsetzung des Prinzips des lebenslangen Sprachenlernens. EPOS kann sehr einfach von einer zur nächsten Bildungsstufe „mitgenommen“ werden, von der Schule zur Universität oder Berufsausbildung, zur Weiterbildung in der beruflichen (Sprach-) Praxis oder zur Unterstützung privater Interessen an sprachlicher und interkultureller Bildung. ${ }^{9}$

\subsubsection{Einsatzszenarien}

Die geschilderten technischen Möglichkeiten entfalten aber nur dann ihre Wirksamkeit, wenn ihnen ein sinnvolles didaktisches Konzept zugrunde liegt. Solche Konzepte sollten vor dem Einsatz von EPOS curricular ausgearbeitet und validiert werden. Dabei sollten die einzusetzenden EPOS-Funktionen passgenau bestimmt und das System entsprechend konfiguriert werden, um jede Form von technischem Overkill zu vermeiden. Im Folgenden werden beispielhaft einige Szenarien kurz erläutert.

\footnotetext{
${ }^{9}$ Mit der Exportfunktion erfüllt EPOS u.a. auch eine Anforderung aus der Europäischen Datenschutzgrundverordnung, die den Betroffenen ein „Recht auf Datenübertragbarkeit“ einräumt (Art. 20 DSGVO).
} 


\section{Tutorenprogramm}

Am Fremdsprachenzentrum der Hochschulen im Land Bremen wird EPOS zur Unterstützung in der Sprachlernberatung eingesetzt (vgl. Buschmann-Göbels et al. 2015, Buschmann-Göbels, Jahnke 2017). Angesichts einer zunehmend heterogeneren Studierendenschaft wird Lernberatung immer wichtiger, insbesondere in kursunabhängigen Lernszenarien. Eine Form der Lernberatung stellt das Tutorenprogramm dar. Hier lernen die Studierenden die jeweilige Zielsprache im Rahmen eines selbst gewählten Projekts und auf ein bestimmtes Lernziel hin. Sie arbeiten in kleinen Gruppen zusammen und werden dabei von ausgebildeten studentischen Tutor*innen betreut. Ein didaktisches Element des Tutorenprogramms ist der Einsatz von EPOS, neben anderen, wie Lernziel- und Lernwegplanung, Projektarbeit und Zeitmanagement. Die Studierenden nutzen EPOS, um ihre Sprachkenntnisse zu Beginn des Programms selbst einzuschätzen, um Lernergebnisse im Dossier zu dokumentieren und im Lerntagebuch zu reflektieren, aber auch um mit der vorhandenen Gruppenfunktion kollaborativ zu lernen. Die Tutor*innen ihrerseits arbeiten ebenfalls mit EPOS, z.B. um den Studierenden im Rahmen der Beratung - die face-to-face, aber auch elektronisch stattfindet - Feedback zu ihren Artefakten (Texte, Audiodateien, Videos) zu geben sowie um ihren eigenen Beratungsprozess zu reflektieren.

\section{Tandem}

An der Universität Paderborn wird EPOS zur Unterstützung des Tandemlernens eingesetzt (vgl. Dönhoff 2015). Bei dem von Ilka Dönhoff beschriebenen Szenario werden im Sinne eines, angepassten ePortfolios“ nur ausgewählte EPOSFunktionen genutzt, und zwar die Selbsteinschätzung, die Festlegung der Lernziele, die Erstellung einer Ansicht und die Benutzung des Forums. Die Nutzung des Dossiers war freigestellt (es konnte das EPOS-Dossier oder ein Papierdossier/eine Sammelmappe genutzt werden), das Lerntagebuch und die Lernbiographie wurden nicht benutzt. In einem anderen Szenario von Dönhoff wurde aber für die Reflexion das Lerntagebuch sehr wohl eingesetzt und dabei durch ausführliche Leitfragen und Materialverlinkung eine intensive Anregung für die Reflexion der Tandemarbeit gegeben. Die Konfigurierbarkeit von EPOS stellt in diesen Szenarien einen großen Vorteil dar. 


\subsection{Lessons learnt}

EPOS ist seit 2013 bei einer Reihe in- und ausländischer Hochschulen im Einsatz. Sie tauschen ihre Erfahrungen aus. Einige von ihnen führten bereits Evaluierungen mit Studierenden und Lehrenden durch. Im Folgenden werden einige der wesentlichen Ergebnisse sowie die nächsten Entwicklungsschritte kurz zusammengefasst. ${ }^{10}$

EPOS wird von den Lernenden und Lehrenden grundsätzlich positiv beurteilt. Folgende Vorteile werden durchgängig genannt. Gegenüber einem Papierportfolio bieten ePortfolios die Möglichkeit zur Einbeziehung von Dokumenten nicht-schriftlicher Art. Während die klassische Sammelmappe nur schriftliche Texte enthält, können nunmehr auch mündliche Dokumente (Audiodateien), teilweise kombiniert mit non-verbalen Kommunikationselementen (Videodateien) erstellt, gespeichert, beurteilt und reflektiert werden. Dies wirkt der bisher ohnehin stark schriftlastigen Ausrichtung der Sprachlehre entgegen. Gleichzeitig wird die dynamische Seite von ePortfolios hervorgehoben: Selbsteinschätzungen, die zu verschiedenen Zeitpunkten erstellt wurden, können leicht miteinander verglichen und damit der Lernfortschritt anschaulich sichtbar gemacht werden. Die Möglichkeit zur durchgängigen Nutzung von Portfolios über Bildungsstufen hinweg und idealerweise lebenslang wird erst in ePortfolios möglich. Auch die einfache Möglichkeit, Elemente des Lernprozesses (Selbsteinschätzungen, Artefakte, Reflexionen usw.) für andere nicht nur Lehrenden, sondern auch Peers - differenziert frei zu geben, stellt insbesondere aus Sicht der Lehrenden einen nicht unwesentlichen didaktischen Vorteil dar. Die Studierenden schätzen die Möglichkeit, Ansichten kreativ gestalten und sich dabei multimedialer Elemente bedienen zu können. Wenn diese Möglichkeiten in Projekte eingebettet sind, die in EPOS organisiert werden können, ist das Feedback der Studierenden besonders positiv.

In der Praxis werden nicht alle diese Vorteile tatsächlich auch genutzt. Es werden immer noch meist schriftliche Dokumente verwendet, sei es, dass sich die Anforderungen der Lehrenden noch nicht auf das neue Medium eingestellt haben, sei es, dass den Lernenden die Produktion und das Hochladen von Audio- oder Videodateien (noch) zu kompliziert erscheint. Die Nutzung von EPOS erfordert eine Einarbeitung in das System. Den Studierenden stellt sich die Frage, ob der Nutzen des Einsatzes in nur einer oder wenigen Veranstaltungen im Vergleich zum Aufwand angemessen ist. Hier würde das Konzept einer Veranstaltungs- oder auch Semester-übergreifenden Nutzung des ePortfolio-Systems die Aufwand-NutzenRelation positiv beeinflussen. Insgesamt lässt sich die Beurteilung des Einsatzes von ePortfolios und damit auch von EPOS folgendermaßen zusammenfassen:

Erstens, die positiven bzw. zustimmenden Aussagen zum Einsatz von EPOS überwiegen. EPOS wird gegenüber dem Papierportfolio deutlich bevorzugt.

\footnotetext{
102018 war EPOS bei etwa 15 Hochschulen aus Deutschland, England, Frankreich, Schweiz und Japan im Einsatz. Die Evaluationsergebnisse stammen im Wesentlichen von Universitäten in Bochum, Bremen, London, Paderborn und Paris.
} 
Studierende loben die Interaktivität und Multimedialität von EPOS, oder wie es eine Bochumer Studentin formulierte: „Mehr EPOS statt Hausaufgaben.“

Zweitens, die Benutzbarkeit von EPOS muss weiter verbessert werden (Reduzierung der Komplexität). EPOS muss curricular im Sinne des Blended Learning so in Lehrveranstaltungen oder andere Lernkontexte eingebunden werden, dass seine Nutzung für die Studierenden inhaltlich und formal motivierend wirkt. Ein gutes Beispiel dafür ist die Nutzung von EPOS im Rahmen von Lernprojekten.

\section{Literatur}

Brunner, Ilse; Häcker, Thomas; Winter, Felix (Hrsg.) (2008): Das Handbuch Portfolioarbeit. Konzepte, Anregungen, Erfahrungen aus Schule und Lehrerbildung. SeelzeVelber: Klett Kallmeyer.

Buschmann-Göbels, Astrid; Bornickel, Marie; Nijnikova, Marina (2015): Meet the Needs - Lernberatung und tutorielle Lernbegleitung heterogener Lerngruppen zwischen individuellen Bedürfnissen und fachlichen Anforderungen. In: Zeitschrift für interkulturellen Fremdsprachenunterricht 20/1, 181-200. http:/ / tujournals. ulb.tu-darmstadt.de/index.php/zif/article/view/197/190 [15.03.2018].

Buschmann-Göbels, Astrid; Jahnke, Annette (2017): Meet the Needs. Lernberatung heterogener Lerngruppen zwischen individuellen Bedürfnissen und fachlichen Anforderungen. In: Böcker, Jessica; Saunders, Constanze; Koch, Lennart; Langner, Michael (Hrsg.): Beratung und Coaching zum Fremdsprachenlernen. Gießen: Giessener Elektronische Bibliothek. http://geb.uni-giessen.de/geb/volltexte/ 2017/12596/pdf/GiFon_9.pdf [15.03.2018].

Comenius, Johann Amos (2008): Große Didaktik: Die vollständige Kunst, alle Menschen alles zu lehren. Übersetzt und herausgegeben von Andreas Flitner. 10. Aufl. Stuttgart: Klett-Cotta. (Original Didactica Magna, Amsterdam 1657).

Council of Europe (o,J.): Accredited and Registered ELP. https://www.coe.int/en/ web/portfolio/accredited-and-registered-elp [01.06.2018].

Council of Europe (1992): Transparency and Coherence in Language Learning in Europe: Objectives, Evaluation, Certification. Report on the Rüschlikon Symposium. Strasbourg: Council of Europe.

Council of Europe (2011): European Language Portfolio. Principles and Guidelines.

(Version 2). Strasbourg: Council of Europe. Language Policy Division (Original October 2000, revised June 2004; January 2011).

Council of Europe (2006): European Language Portfolio: Key Reference Documents. Strasbourg: Council of Europe.

Council of Europe (2018a): Common European Framework of Reference for Lan185anges: Learning, Teaching, Assessment - Companion Volume with New Descriptors. Strasbourg: Council of Europe, Language Policy Programme. 
Council of Europe (2018b): Reference Framework of Competences for Democratic Culture. Vol. 1-3. Strasbourg: Council of Europe Publishing.

Dewey, John (1916): Democracy and Education. New York: Macmillan.

Dönhoff, Ilka (2015): Das digitale Dossier im Sprachtandem - Ergebnisse einer empirischen Pilotstudie zum ePortfolio EPOS. In: die hochschullebre - Interdisziplinäre Zeitschrift für Studium und Lehre 1, 1-18. http:/ /www.hochschullehre.org/wpcontent/files/doenhoff_2015.pdf [15.03.2018].

Egle, Gert (2015): Portfoliotypen. In: teachSam - Bildungsserver. http://www.teachsam.de/arb/portfolio/portfolio_3.htm [01.06.2018].

Emmett, David J. (2011): Student Engagement with an ePortfolio: A Case Study of Preservice Education Students. Dissertation, Queensland University of Technology, Faculty of Education. https://eprints.qut.edu.au/40957/1/David _Emmett_Thesis.pdf [01.06.2018].

Europarat (2001): Gemeinsamer europäischer Referenzrahmen für Sprachen: lernen, lehren, beurteilen. München: Klett-Langenscheidt.

Europarat (2018): Kompetenzen für eine demokratische Kultur- Gleichberechtigtes Zusammenleben in kulturell unterschiedlichen demokratischen Gesellschaften. Strasbourg: Council of Europe Publishing.

Fehse, Rüdiger; Friedrich, Jürgen; Kühn, Bärbel (2011): EPOS - An ePortfolio for language learning. In: Proceedings of the $4^{\text {th }}$ International Conference "TCT for Language Learning”. Florenz. http:/ /www.sprachenrat.bremen.de/files/Literatur/ILT66-445ABS-Fehse-ICT4LL2011.pdf [01.06.2018].

Häcker, Thomas (2011): Portfolioarbeit - Ein Konzept zur Wiedergewinnung der Leistungsbeurteilung für die pädagogische Aufgabe der Schule. In: Sacher, Werner; Winter, Felix (Hrsg.): Diagnose und Beurteilung von Schülerleistungen. Grundlagen und Reformansätze. Baltmannsweiler: Schneider Hohengehren, 217-230.

Holec, Henri (1981): Autonomy and Foreign Language Learning. Oxford, New York: Pergamon Press.

Little, David (1991): Learner Autonomy. 1: Definitions, Issues and Problems. Dublin: Authentik.

Little, David (2007): Language Learner Autonomy: Some Fundamental Considerations Revisited. In: Innovation in Language Learning and Teaching 1/1, 14-29.

Little, David (2012): The European Language Portfolio - History, key concerns, future prospects. In: Kühn, Bärbel; Pérez Cavana, María Luisa (Hrsg.): Perspectives from the European Language Porfolio. Learner Autonomy and SelfAssessment. London, New York: Routledge, 7-21.

Reich, Kersten (o.J.): Portfolio. In: Reich, Kersten. (Hrsg.): Methodenpool. Universität zu Köln. http://methodenpool.uni-koeln.de [01.06.2018]. 
Reinders, Hayo (o.J.): Autonomy Bibliography. https://innovationinteaching.org/freetools/autonomy-bibliography.php [04.04.2019].

Sudhershan, Aleksandra (2014): E-Portfolio self-assessment of intercultural communicative competence: Helping language learners to become autonomous intercultural speakers. In: Geraghty, Barbara; Conacher, Jean E. (Hrsg.): Intercultural Contact, Language Learning and Migration. London, New York:

Bloomsbury Publishing, 150-165.

Tassinari, Maria Giovanna (2010): Autonomes Fremdsprachenlernen. Komponenten, Kompetenzen, Strategien. Frankfurt/M.: Lang. 